\title{
Urbanorum spp. EN EL PERÚ Urbanorum spp. IN PERU
}

\author{
Ruth Inés Mirano Villafuerte ${ }^{1, a}$, Luis Antonio Zapata Collado ${ }^{1, a}$, César Náquira Velarde $2, b$
}

El investigador Francisco Tirado Santamaría, catedrático de parasitología de la Universidad Industrial de Santander (UIS), describió a este aparente microorganismo como un protozoo, similar a las amebas, presente en las heces de seres humanos y con características particulares lo cual le permite nominarlo Urbanorum spp. (1). En 1991 se le empezó a observar en muestras de pacientes atendidos en el Centro de Salud de Barrancabermeja, se trata de estructuras redondeadas de entre 80 y 100 $\mu$ de diámetro y que teñido con lugol se observa un contenido de color amarillo claro y una doble membrana externa que presenta poros a través de los cuales estructuras hialinas emergen de su interior, semejantes a seudópodos. Su reproducción parece ser por división binaria, por lo que se le considera como protozoo ${ }^{(2)}$.

Los pacientes que tienen Urbanorum spp. manifiestan presentar unas deposiciones diarreicas, sin moco, sangre o leucocitos, y dolor tipo cólico en su fase inicial en el hipocondrio derecho y parte baja del abdomen que sugiere, hipotéticamente, que el proceso es en el colon ${ }^{(1)}$. En 1996, Tirado Santamaría encontró el microorganismo en el 13,98\% de 143 muestras analizadas; en un estudio más amplio entre el año 1997 y 1998, se analizaron 14000 muestras, encontrándose una prevalencia del $10 \%{ }^{(3)}$. En el año 2006 , en el estudio "Prevalencia de parásitos intestinales en niños en edad escolar de Barrancabermeja, se analizaron 500 muestras y se encontró una prevalencia del 10\% (Muñoz G. et al. 2006) (4). En el año 2007 en el estudio "Prevalencia de parásitos intestinales en niños en edad escolar en Piedecuesta- Santander" se analizaron 200 muestras de materia fecal, encontrándose un $5 \%$ de Urbanorum ssp ${ }^{(4)}$

En la presente galería fotográfica se muestran los resultados de una mujer de 67 años de edad, procedente de San Juan de Lurigancho (Lima), que acude a consulta externa de medicina general del Hospital Aurelio Díaz
Ufano y Peral refiriendo que durante unos 9 meses venía presentando dolor abdominal y dispepsia, por lo cual se le solicita exámenes auxiliares de parasitológico seriado y directo además de Thevenon. Las muestras entregadas por la paciente presentaron contextura blanda; luego, mediante el método de concentración por centrifugación ${ }^{(5)}$ pudo evidenciarse la presencia de Urbanorum spp., 2-4 por campo, en 10x de aumento (Figura.1); Blastocystis hominis, 15-17 por campo; Entamoeba coli, 1-3 por campo; lodamoeba butschlii, 0-2 por campo; no se observó leucocitos ni hematíes, y el Thevenon fue negativo.

En la presente galería se puede apreciar al Urbanorum spp. en sus diversas formas en las muestras de heces figuras ${ }^{(2,3,4,5,6,7)}$. El presente hallazgo en la paciente, es similar al descrito en la información original de Francisco Tirado Santamaría. La literatura muestra la presencia de este parásito en Colombia, asimismo, se requiere más estudios de la morfología, fisiología, ciclo biológico, y epidemiología que permita ratificar o rectificar la ubicación taxonómica del Urbanorum spp.

En el caso que reportado la paciente no solo era portadora de Urbanorum spp., sino también otros protozoos que se adquieren por fecalismo, lo cual podría indicar que es por semejante mecanismo que se adquiere la infección de Urbanorum spp.

Fuentes de financiamiento: autofinanciado.

Conflictos de interés: los autores declaran no tener conflictos de interés en la publicación de este artículo.

Contribuciones de los autores: RIMV ha participado en la concepción del artículo, redacción del artículo, procesamiento de muestras; LAZC ha participado en la recolección de los datos y procesamiento de muestras. CGNV ha contribuido en la revisión crítica del artículo, como asesor científico de la presente galería.

\footnotetext{
Hospital I Aurelio Díaz Ufano y Peral. Lima, Perú.

Instituto de Medicina Tropical Daniel A. Carrión, Universidad Nacional Mayor de San Marcos. Lima, Perú.

a Tecnólogo médico; ${ }^{b}$ médico cirujano especialista en Parasitología Humana, doctor en Medicina

Recibido: 02/04/2016 Aprobado: 20/04/2016
}

Citar como: Mirano Villafuerte RI, Zapata Collado LA, Náquira Velarde C. Urbanorum spp. en el Perú. Rev Peru Med Exp Salud Publica. 2016;33(3):593-5. doi: http://dx.doi.org/10.17843/rpmesp.2016.333.2351 


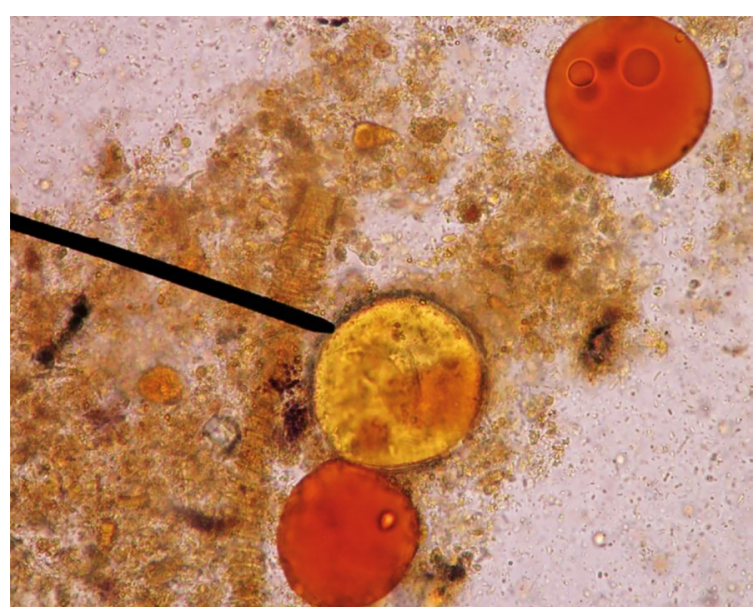

Figura 1. Urbanorum spp. en lugol 10x; sin pseudópodos, asemejando gotas de grasa

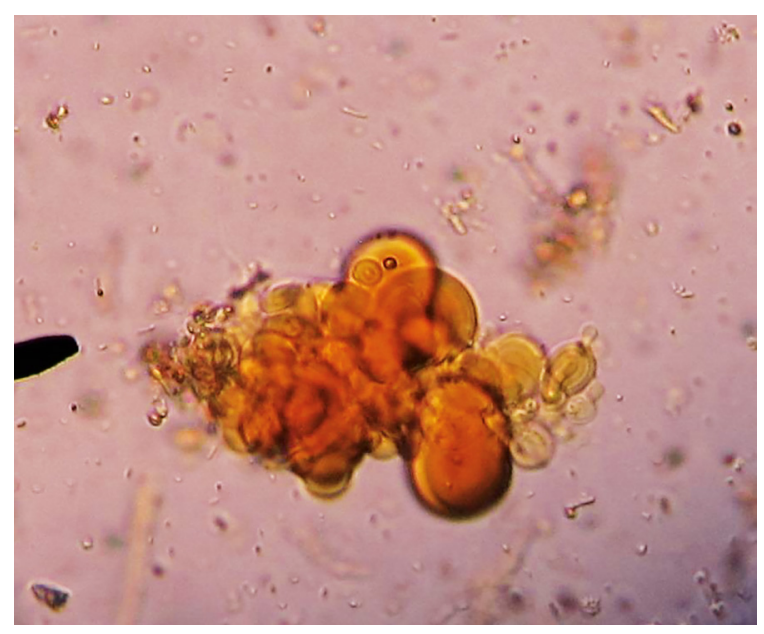

Figura 3. Urbanorum spp. en lugol 10x

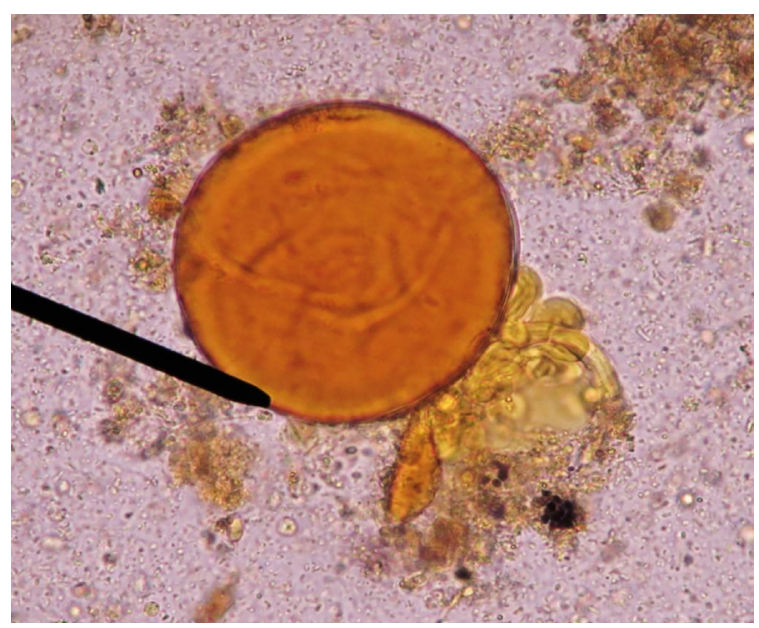

Figura 5. Urbanorum spp. En lugol 40x, con pseudópodos

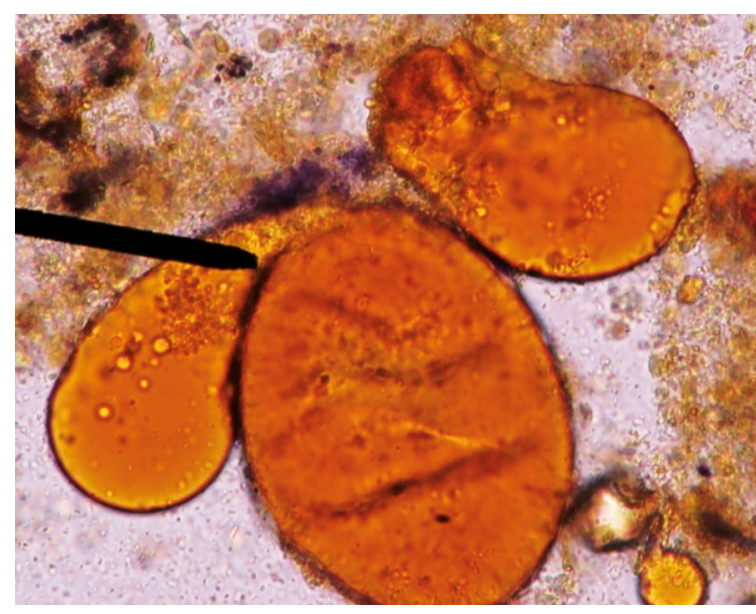

Figura 2. Urbanorum spp. en lugol 40x

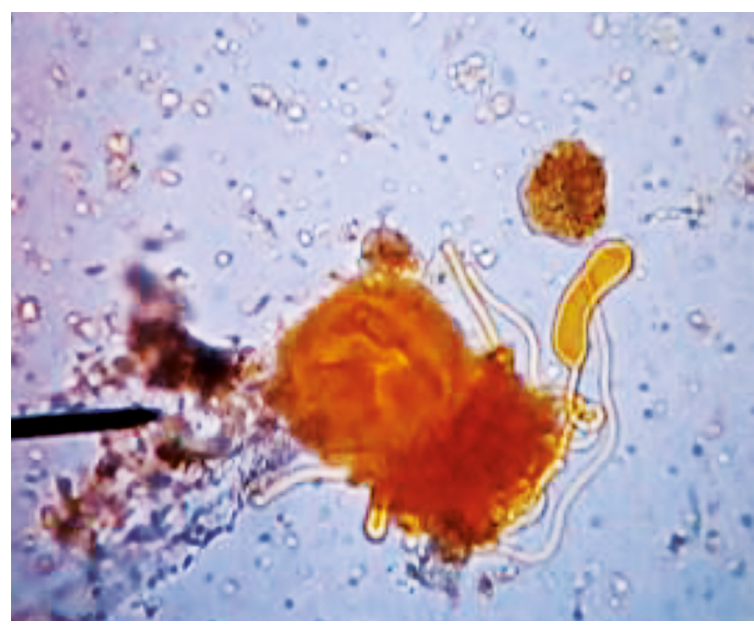

Figura 4. Urbanorum spp. en lugol 10x, con pseudópodos

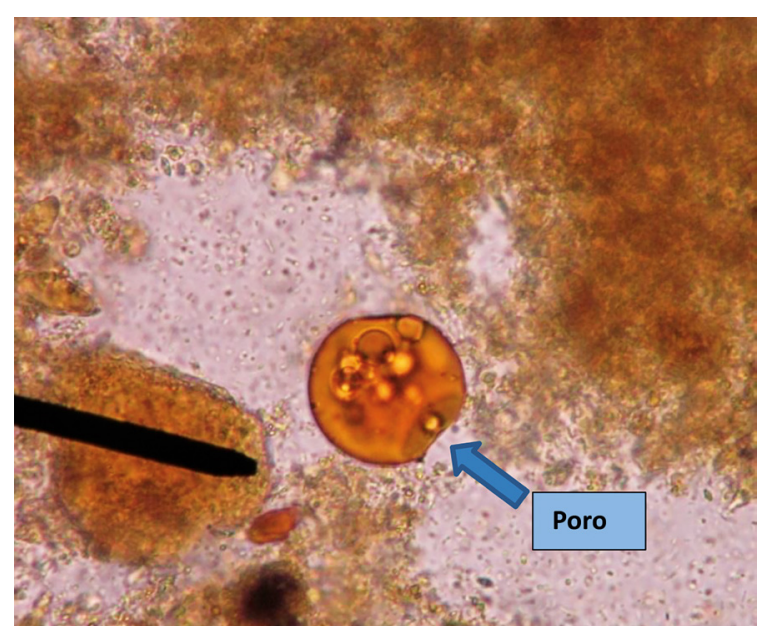

Figura 6. Urbanorum spp. en lugol 10x, poro de salida de sus pseudópodos 


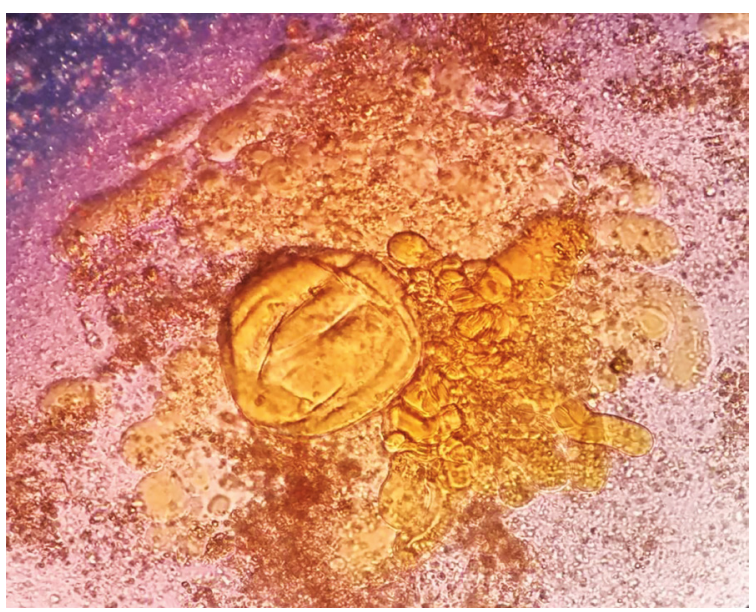

Figura 7. Urbanorum spp. en lugol 10x, con pseudópodos

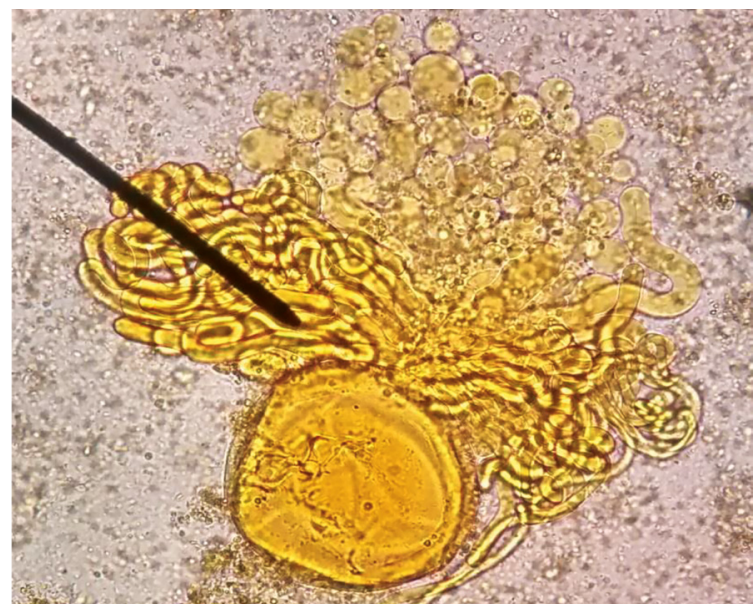

\section{REFERENCIAS BIBLIOGRÁFICAS}

1. Francisco Tirado Santamaría. Urbanorum spp. [internet]. Santander: Catedra Libre UIS; 2013 [citado el 30 de julio de 2015]. Disponible en: http://www.buenastareas.com/ ensayos/Urbanorum-Spp/70918639. html

2. Abarca C, Córdova D, Jaramillo J, Oyagata J, Tenesaca J. Urbanorum spp. Parásito intestinal [internet]. Santander: Catedra Libre UIS; 2015 [citado el 30 de julio de 2015]. Disponible en: https://prezi.com/ wfrtxkhkdrwn/urbanorum-spp/
3. Jaramillo-Areiza AL. Urbanorum spp. ¿Nuevo parásito intestinal? Ahora en Antioquia [internet]. Antioquia: 2014, May. [citado el 30 de julio de 2015]. Disponible en: http:// es.slideshare.net/anyijaramillo19/ u r b a n or u m - s p p - c a s o clnico-341679851.-

4. Infiobioquimica. (2008). Nuevo parásito intestinal descubierto en Santander. (Disponible en http:// www.infobioquimica.com/index. $\mathrm{php/home/noticias-eventos/30-}$ noticias/1515-nuevo-parasito- intestinal-descubierto-en-santander. Consultado el 30 de julio de 2015.

5. Craig y Faust. Parasitología Clínica. Barcelona: El Salvat; 1975.
Correspondencia: Ruth Ines Mirano Villafuerte Dirección: MZ J LT 19 Grupo 1 Cruz de Motupe San Juan de Lurigancho. Lima, Perú. Teléfono: 956356925

Correoelectrónico: ruthynes18@gmail.com

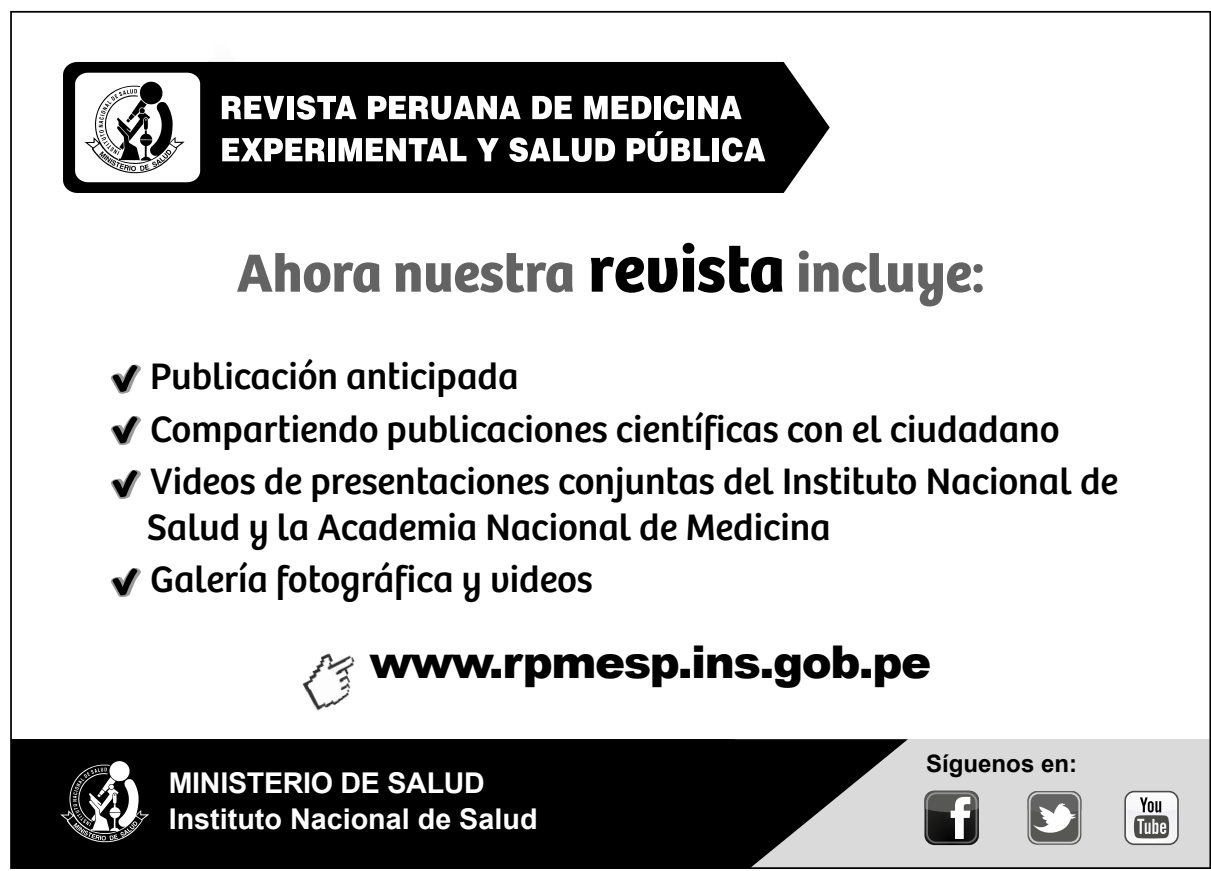

